jet Enterprise 7000n. Das Gerät (Preis rund 3.200 Euro) verfügt über ein Farbdisplay mit Touchscreen zum Anzeigen, Prüfen und Ändern gescannter Dokumente. _Direktscannen in E-Mails. Dies ist eine Funktion, die für Praxen hilfreich ist, in denen die Helferinnen oft an Arztbriefe oder andere Korrespondenz häufig gescannte Dokumente anhängen müssen oder wenn Mediziner in Ärztenetzen schnell mit Kollegen oder anderen Praxen kommunizieren wollen. Multifunktionsgeräte wie der Lexmark X204n (Preis 299 Euro) können oft auch direktscannen.

_Sollen die gescannten Dokumente an verschiedenen Rechnern bearbeitet und verwaltet werden oder soll der Scanner mit verschiedenen Rechnern kommunizieren können, sollte ein Gerät mit Netzwerkfunktion gewählt werden. Ein Beispiel ist der Epson GT-30000, der jedoch preislich bei über 4.300 Euro liegt.

Für Praxen ist es wichtig, dass die häufig textlastigen Dokumente in der Praxis-EDV auch weiterverarbeitet werden können. Deshalb brauchen sie zusätzlich eine sogenannte OCRSoftware (siehe Kasten). Diese wandelt die gescannten in bearbeitbare Dokumente um beziehungsweise konvertiert sie in PDF- oder Textdateien wie zum Beispiel doc. Dabei gibt es Geräte - meist aus dem hochpreisigeren Segment - die bereits die Software mitliefern. Für einen Preis von rund 100 Euro lässt sich aber etwa mit OmniPage 18 Standard bereits eine leistungsfähige Software erwerben, die auch Spalten, Tabellen und Grafiken gescannter Dokumente gut erfasst. Dabei kann die Software auch per Digitalkamera, iPhone oder iPad abfotografierte Dokumente umwandeln. Bei vielen OCR-Programmen, etwa dem FineReader11 (Preis für die Professional Edition 129 Euro) können die gescannten Dokumente auch direkt an Anwendungen wie Microsoft Word, Excel und PowerPoint oder Adobe Acrobat gesendet werden. RebekkaHöhl

\title{
Krankenschwester aus dem PC kommt gut an
}

Um den Pflegenotstand etwas abzufedern, haben US-Forscher der Northeastern University in Chicago eine virtuelle Krankenschwester namens "Elizabeth" entwickelt. Elizabeth komme sehr gut bei den Patienten an, heisst es in einem Bericht des Magazins Technology Review. Bereits nach der ersten Studie der Northeastern University mit Elizabeth hätten Patienten angegeben, dass sie die virtuelle Schwester menschlichem Klinikpersonal vorzögen. Noch interessanter ist aber, dass Patienten, die durch das Programm betreut wurden, laut der Forscher nach der Klinikentlassung eher zu Folgeuntersuchungen gingen und besser über ihre Krankheit Bescheid wussten als Patienten, die nur durch echtes Pflegepersonal betreut wurden. 